DOI $10.1007 / \mathrm{s} 10958-020-04835-9$

Journal of Mathematical Sciences, Vol. 247, No. 5, June, 2020

\title{
EXTREMAL AREAS OF POLYGONS WITH FIXED PERIMETER
}

\author{
G. Khimshiashvili, ${ }^{*}$ G. Panina, ${ }^{\dagger}$ and D. Siersma ${ }^{\ddagger}$ \\ UDC 515.164.174 \\ We consider the configuration space of planar n-gons with fixed perimeter, which is diffeomorphic \\ to the complex projective space $\mathbb{C} P^{n-2}$. The oriented area function has the minimum number of \\ critical points on the configuration space. We describe its critical points (these are regular stars) \\ and compute their indices when they are Morse. Bibliography: 11 titles.
}

\section{INTRODUCTION}

One of the results on the isoperimetric problem discussed in the classical treatise by Legendre [7] states that the regular $n$-gon has the maximum area among all $n$-gons with fixed perimeter. For the historic development of this problem, we refer to [2]. The aim of the present paper is to elaborate upon this classical result by placing it in the context of Morse theory on a naturally associated configuration space. To this end, we follow the paradigms used in $[9,10]$ and begin with several definitions and recollections.

An $n$-gon is an $n$-tuple of points $\left(p_{1}, \ldots, p_{n}\right) \in\left(\mathbb{R}^{2}\right)^{n}$, some of which may coincide. Its perimeter is (as usual)

$$
\mathcal{P}\left(p_{1}, \ldots, p_{n}\right)=\left|p_{1} p_{2}\right|+\left|p_{2} p_{3}\right|+\cdots+\left|p_{n} p_{1}\right| .
$$

The configuration space $\mathcal{C}_{n}$ considered in what follows is defined as the space of all polygons (modulo rotations and translations) whose perimeter equals 1 (one can fix any other positive number).

The oriented area of a polygon with vertices $p_{i}=\left(x_{i}, y_{i}\right)$ is defined as

$$
2 A=x_{1} y_{2}-x_{2} y_{1}+\cdots+x_{n} y_{1}-x_{1} y_{n} .
$$

The oriented area as a Morse function has been studied in various settings: for configuration spaces of flexible polygons (those with the side lengths fixed) in $\mathbb{R}^{2}$ and $\mathbb{R}^{3}$, see $[5,9-11]$. We shall use some of the previous results in the new setting of this paper.

Let $\sigma$ be the cyclic renumbering: given a polygon $P=\left(p_{1}, \ldots, p_{n}\right)$,

$$
\sigma\left(p_{1}, \ldots, p_{n}\right)=\left(p_{2}, p_{3}, \ldots, p_{n}, p_{1}\right) .
$$

In other words, we have an action of $\mathbb{Z}_{n}$ on $\mathcal{C}_{n}$ which renumbers the vertices of a polygon.

A regular star is an equilateral $n$-gon such that

$$
\sigma\left(p_{1}, \ldots, p_{n}\right)=\left(p_{1}, \ldots, p_{n}\right),
$$

see Figs. 1, 2.

A complete fold is a regular star with $p_{i}=p_{i+2}$. It exists for even $n$ only.

A regular star that is not a complete fold is uniquely determined by its winding number $w$ with respect to the center.

We can now formulate the main result of the paper.

\footnotetext{
${ }^{*}$ Ilia State University, Tbilisi, Georgia, e-mail: gogikhim@yahoo.com.

${ }^{\dagger}$ St.Petersburg Department of Steklov Institute of Mathematics and St.Petersburg State University, St.Petersburg, Russia, e-mail: gaiane-panina@rambler.ru.

${ }^{\ddagger}$ Utrecht University, Utrecht, The Netherlands, e-mail: d.siersma@uu.nl.
}

Published in Zapiski Nauchnykh Seminarov POMI, Vol. 481, 2019, pp. 136-145. Original article submitted Jule 12, 2019. 
Theorem 1. (1) The space $\mathcal{C}_{n}$ is homeomorphic to $\mathbb{C} P^{n-2}$. Therefore, we regard it as a smooth manifold, keeping in mind the smooth structure of the projective space.

(2) Smooth critical points of the function $A$ on $\mathcal{C}_{n}$ are regular stars and complete folds only.

(3) Nonsmooth points of $A$ are Lipschitz-regular points of $A$.

(4) The function $A$ has the minimum number of critical points on $\mathbb{C} P^{n-2}$.

(5) The stars with the maximum winding numbers are nondegenerate critical points of the function A.

(6) Under the assumption that all regular stars are nondegenerate critical points, the Morse index is

$$
M(P)= \begin{cases}2 w(P)-2 & \text { if } w(P)<0 \\ 2 n-2 w(P)-2 & \text { if } w(P)>0 \\ n-2 & \text { if } P \text { is a complete fold }\end{cases}
$$

As an illustration, below we present figures showing some regular stars together with their winding numbers.

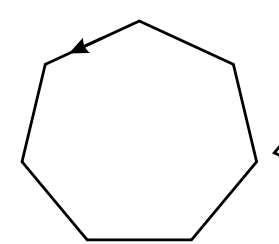

$w=1$

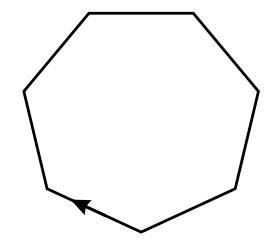

$w=-1$

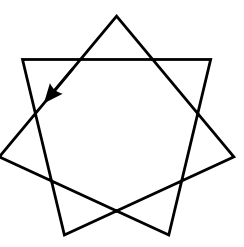

$\mathrm{w}=2$

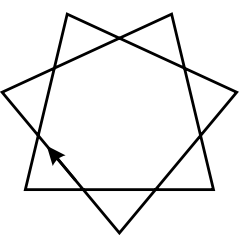

$w=-2$

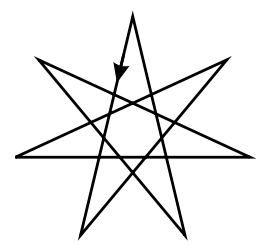

$w=3$

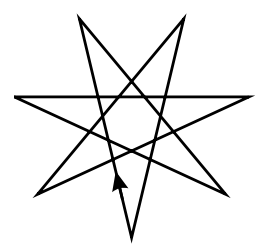

$w=-3$

Fig. 1. Regular stars for $n=7$ with their winding numbers.

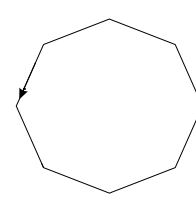

$w=1$

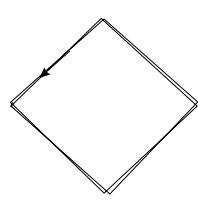

$\mathrm{w}=2$

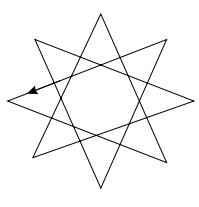

$w=3$

complete fold

Fig. 2. Regular stars for $n=8$ with positive winding numbers.

It is worth mentioning that a scholarly example of an exact Morse function on the complex projective space is

$$
F\left(u_{1}: \cdots: u_{n-1}\right)=\frac{a_{1}\left|u_{1}\right|^{2}+\cdots+a_{n}\left|u_{n-1}\right|^{2}}{\left|u_{1}\right|^{2}+\cdots+\left|u_{n-1}\right|^{2}}
$$

for distinct real numbers $a_{i}$. We will discuss more on this in the last section; now let us only mention that an obvious difference with the area function is that a cyclic permutation of indices preserves the critical points of the area and cyclically permutes the critical points of $F$. 


\section{Proof of TheOREM 1}

There are three (well-known) statements to be used in the proof.

Statement A. Assume that a smooth manifold $C^{\prime}$ is a codimension $k$ submanifold of a smooth manifold $C$. Let $q \in C^{\prime}$ be a Morse point of some function $f: C \rightarrow \mathbb{R}$. If $q$ is also a Morse point of the restriction $\left.f\right|_{C^{\prime}}$, then for the Morse indices of $f$ related to $C$ and $C^{\prime}$, we have

$$
M_{C^{\prime}}(q)+k \geq M_{C}(q) \geq M_{C^{\prime}}(q) .
$$

The proof easily follows from Sylvester's formula for the index of a symmetric matrix.

Statement B. Assume that a smooth manifold $C$ is a Cartesian product $C=C_{1} \times C_{2}$, and a function $f: C \rightarrow \mathbb{R}$ is a sum $f=f_{1}+f_{2}$ where $f_{1,2}: C_{1,2} \rightarrow \mathbb{R}$. Then $q=\left(q_{1}, q_{2}\right) \in C$ is a Morse point if and only if $q_{1}$ and $q_{2}$ are Morse points of $f_{1}$ and $f_{2}$, respectively. In this case, the Morse indices sum up:

$$
M(q)_{C}=M_{C_{1}}\left(q_{1}\right)+M_{C_{2}}\left(q_{2}\right) .
$$

This follows by noticing that, in this case, the Hessian matrix has block form.

Statement C. Assume that $q$ is a point in a smooth manifold $M^{n}$ that is an isolated critical point of a smooth function $f$. Assume that there exist two submanifolds of complementary dimensions $N^{k} \subset M^{n}$ and $L^{n-k} \subset M^{n}$ intersecting transversally at $q$. Assume also that $q$ is a nondegenerate local maximum of the restriction $\left.f\right|_{N^{k}}$ and a nondegenerate local minimum of the restriction $\left.f\right|_{L^{n-k}}$. Then $q$ is a Morse point of $f$ whose Morse index is $k$.

The proof again follows from Sylvester's formula for the index of a symmetric matrix.

Claim (1) of Theorem 1 was probably known earlier. Assume that we have a polygon $P \in \mathcal{C}_{n}$. Identifying its ambient space $\mathbb{R}^{2}$ with $\mathbb{C}$, let us interpret its edges $p_{1} p_{2}, \ldots, p_{n-2} p_{n-1}$ as complex numbers $u_{1}, \ldots, u_{n-1}$. This $(n-1)$-tuple is never identically zero, so it determines a point $\left(u_{1}: \cdots: u_{n-1}\right) \in \mathbb{C} P^{n-2}$. Conversely, each point in the projective space yields a set of mutually homothetic polygons with a unique representative whose perimeter equals 1 .

Claim (2) appeared already in [6] (yet is unpublished). The proof follows from two observations.

First, it is easy to show that a critical point is an equilateral polygon. Second, freeze the points $p_{1}, p_{3}, \ldots, p_{n}$. Setting $l_{1}=\left|p_{1} p_{2}\right|, l_{2}=\left|p_{2} p_{3}\right|$, move the point $p_{2}$ in such a way that $l_{1}+l_{2}$ remains fixed. The point $p_{2}$ travels along an ellipse. The area of the triangle $p_{1} p_{2} p_{3}$ is critical if and only if $l_{1}=l_{2}$.

Second, if we freeze all the edge lengths of the polygon, we know from [9] that being critical means being cyclic. Therefore, a critical polygon is an equilateral polygon which is inscribed in a circle.

It remains to consider polygons having coinciding edges going in opposite directions, that is, with $p_{i}=p_{i+2}$. These are complete folds. Claim (2) is proven.

Claim (3) is missing in [6]. The configuration space $\mathcal{C}_{n}$ inherits a smooth structure from $\mathbb{C} P^{n-2}$, but the function $A$ is not everywhere continuously differentiable, since the perimeter involves square roots. The nonsmooth points are the configurations with two (or more) colliding consecutive vertices; they form an arrangement of hyperplanes in $\mathbb{C} P^{n-2}$. To make sure that nonsmooth points of $A$ "behave like regular ones" in the Morse-theoretic sense, we will use the concept of Clarke subdifferential $\delta_{c}$ from nonsmooth analysis. This subdifferential is related to the limits of all gradients at smooth points in a neighborhood of singular points. It was introduced by Clarke in [4]. The generalized gradient $\delta_{c} f$ of a locally Lipschitz function $f$ has values in the set of nonempty compact convex sets, satisfies the sum, product, quotient, and chain rules with set addition and scalar multiplication. We use especially the paper [1], which contains also the definition of a critical point and the regular interval theorem. 
In the homogeneous coordinates $\left(u_{1}: \cdots: u_{n-1}\right) \in \mathbb{C} P^{n-2}$, the signed area function is $\frac{A}{\mathcal{P}^{2}}$, where $\mathrm{A}$ is the area on $\mathbb{C}^{n-1}$ and $\mathcal{P}$ is the perimeter. More precisely,

$$
A_{\mathbb{C} P^{n-2}}\left(u_{1}: \cdots: u_{n-1}\right)=\frac{\sum_{1 \leq i<j \leq n-1}\left(\overline{u_{i}} u_{j}-\overline{u_{j}} u_{i}\right)}{\left(\left|u_{1}\right|+\cdots+\left|u_{n-1}\right|+\left|u_{1}+\cdots+u_{n-1}\right|\right)^{2}} .
$$

Fix a nonsmooth point of $P$ and let for this point $u_{1}=x_{1}+i y_{1}=0$ and $w:=u_{2}+\cdots u_{n-1} \neq 0$. Now take a local chart and consider the real partial subderivative with respect to $\left(x_{1}, y_{1}\right)$. We use the following facts:

(a) $A^{\prime}(0)=i \cdot w$ (usual derivative); this is $w$ rotated by $\frac{\pi}{2}$;

(b) $\delta_{c} \sqrt{x_{1}^{2}+y_{1}^{2}}=\mathbb{D}$, where $\mathbb{D}$ is the unit disk in $\mathbb{R}^{2}$;

(c) $\delta_{c} \mathcal{P}(0)=\mathcal{P}^{\prime}(0)=\frac{w}{|w|}+\mathbb{D}$ (Clarke derivative via the sum rule).

Next, we use the quotient rule:

$$
\left(\frac{A}{\mathcal{P}^{2}}\right)^{\prime}=\frac{A^{\prime} \cdot \mathcal{P}-2 A \cdot \mathcal{P}^{\prime}}{\mathcal{P}^{3}}=\mathcal{P}_{0} i \cdot w-2 A_{0} \frac{w}{|w|}-2 A_{0} \mathbb{D} .
$$

According to [1], a point $q$ is called a critical point for a locally Lipschitz function $f: M \rightarrow \mathbb{R}$ on a smooth manifold $M$ if and only if $O \in \delta_{c}(f)(q)$. Proposition 1.2 of [1] extends the first Morse lemma (regular interval theorem) for Clarke regular points to locally Lipschitz functions.

We conclude the proof of (3) by showing that a nonsmooth point of $\mathcal{P}$ is never critical with respect to $\frac{A}{\mathcal{P}^{2}}$. It suffices to show this for the partial subderivative with respect to $\left(x_{1}, y_{1}\right)$.

The condition for a critical point in this case is

$$
\mathcal{P}_{0} i \cdot w-2 A_{0} \frac{w}{|w|} \in 2 A_{0} \mathbb{D} .
$$

By computing the norms of both sides we get $\mathcal{P}_{0}^{2}|w|^{2}+4 A_{0}^{2} \leq 4 A_{0}^{2}$, and this is never the case. Claim (3) is proven.

Claim (4). We conclude that the number of critical points is $n-1$. Since the sum of the Betti numbers and the LS-category of $\mathbb{C} P^{n-2}$ is also equal to $n-1$, the number of critical points is minimal.

Claims (5) and (6). Each of the regular stars (which is not a complete fold) is uniquely determined by its winding number. The latter ranges from 1 to $\left[\frac{n-1}{2}\right]$ and from -1 to $-\left[\frac{n-1}{2}\right]$.

Therefore, the Morse function is exact, and the Morse indices of the critical points are $0,2,4, \ldots, 2 n-4$; it remains to understand which star has which index.

Denote by $S(n, w)$ the $n$-gonal regular star with index $w$.

First, observe that each of the regular stars (with winding number $w$ ) has its symmetric image whose winding number is $-w$.

The only exception is a complete fold, which is symmetric to itself.

Splitting construction. Take a neighborhood of a regular star $P$ with a positive winding number $w$ (which is not a complete fold) and consider two transversally intersecting submanifolds of $\mathcal{C}_{n}$. The first one is the configuration space of equilateral polygons EQUILAT. That is, it consists of all polygons whose edge lengths are equal. Its dimension is $n-3$, and the following is known from [9].

Lemma 1. The Morse index of $P$ related to EQUILAT is $n-1-2 w$.

Therefore, for $S\left(n,\left[\frac{n-1}{2}\right]\right)$ the Morse index of $P$ related to this manifold is 0 . Moreover, from [5] it is known that $P$ is a nondegenerate minimum.

The other manifold $C Y C L$ is the subspace of all polygons that are cyclic. Its dimension is $n-1$. 
Lemma 2. Let $P$ be a regular star that is not a complete fold with $w>0$. Then $P$ is a nondegenerate local maximum on CYCL. So, the Morse index of P related to CYCL is $n-1$.

Proof. The problem is equivalent to the following setting. Take the space of all $n$-gons inscribed in the unit circle and consider the function $A_{\mathbb{C} P^{n-2}}=\frac{A}{\mathcal{P}^{2}}$. It is well defined in a neighborhood of a regular star. It suffices to prove that the regular star with $w>0$ is its local maximum.

Introduce local coordinates by setting $x_{i}$ to be the shift of the $i$ th vertex of the star and $t_{i}=x_{i+1}-x_{i}$. Note that $\sum_{i=1}^{n} x_{i}=2 \pi$ and $\sum_{i=1}^{n} t_{i}=0$. We will compute the 2 -jets $j_{2}$ (Taylor expansions up to order 2). This suffices to find the Morse indices. Computations show that in these coordinates, $j_{2} \widetilde{A}$ is a negative definite quadratic form.

In more detail,

$$
\begin{aligned}
& j_{2} \mathcal{P}\left(t_{1}, \ldots, t_{n}\right)=\sqrt{(2-2 \cos \alpha)} \cdot\left(n-1 / 8 \sum_{i=1}^{n} t_{i}^{2}\right) \\
& j_{2} A\left(t_{1}, \ldots, t_{n}\right)=\frac{\sin \alpha}{2} \cdot\left(2 n-\sum_{i=1}^{n} t_{i}^{2}\right)
\end{aligned}
$$

and

$$
j_{2}\left(2 A / \mathcal{P}^{2}\right)\left(t_{1}, \ldots, t_{n}\right)=\frac{1+\cos \alpha}{8 n^{2} \sin \alpha} \cdot\left(4 n-\sum_{i=1}^{n} t_{i}^{2}\right),
$$

where $\alpha=\frac{2 w \pi}{n}$. Note that since $w>0$, the first factor is always positive and, therefore, the quadratic part is negative definite.

The lemma is proven.

Assume that $n$ is odd. Now, an application of Statement C proves Claims (5) and (6) of Theorem 1 for $S\left(n,\left[\frac{n-1}{2}\right]\right)$. By symmetry, Claims (5) and (6) are also proven for $S\left(n,-\left[\frac{n-1}{2}\right]\right)$.

Proof of Claim (6) for the remaining cases. We now assume that all critical points are nondegenerate. Two mutually symmetric stars have indices $i$ and $n-2-i$, so it suffices to prove (6) for one of them. Besides, by symmetry reasons we immediately conclude that Claim (6) holds for complete folds.

First, we prove the statement for odd $n$. Take all the stars $S(n, w)$ with $\frac{n-1}{2}>w>0$. By Lemma 2, we have $M(S(n, w)) \geq n-1$. So, their indices taken together are

$$
2 n-4,2 n-6, \ldots, n+1 \text {. }
$$

Now let us take all the stars $S(n, w)$ with $\frac{n-1}{2}<w<0$. By symmetry, their indices taken together are $0,2,4, \ldots, n-5$. On the other hand, by Lemma 1 , their indices relative EQUILAT give exactly the same set. This relative index never exceeds the index rel $\mathcal{C}_{n}$, so Claim (6) for odd $n$ is proven.

Next, we prove the statement for even $n$. By a kind of analogy, consider all stars $S(n, w)$ with $w>0$. By Lemma 2, we have $M(S(n, w)) \geq n-1$. So, their indices taken together are $2 n-4,2 n-6, \ldots, n$.

Now let us take all the stars $S(n, w)$ with $w<0$. By symmetry, their indices taken together are $0,2,4, \ldots, n-4$. On the other hand, by Lemma 1, their indices relative EQUILAT give exactly the same set. Since the relative index never exceeds the index rel $\mathcal{C}_{n}$, Claim (6) is proven. We have now completed the proof of Theorem 1. 


\section{Concluding Remarks}

The study of Morse functions on the space $\mathcal{C}_{n}=\mathbb{C} P^{n-2}$ can be related to its Veronese-type embedding into the space HMat of Hermitian matrices. Its image is known (see [3]) to be a taut embedding. It follows that the restriction of almost every $\mathbb{R}$-linear function on HMat to the image of $\mathcal{C}_{n}=\mathbb{C} P^{n-2}$ is an exact Morse function, so it has the minimum number of critical points. The function $F$ mentioned in the introduction is of this type. Our function $A$ is not of this type, since in the coordinates of $\mathcal{C}_{n}=\mathbb{C} P^{n-2}$ it is the quotient of the area and the squared perimeter:

$$
A_{\mathbb{C} P^{n-2}}\left(u_{1}: \cdots: u_{n-1}\right)=\frac{\sum_{1 \leq i<j \leq n-1}\left(\overline{u_{i}} u_{j}-\overline{u_{j}} u_{i}\right)}{\left(\left|u_{1}\right|+\cdots+\left|u_{n-1}\right|+\left|u_{1}+\cdots+u_{n-1}\right|\right)^{2}} .
$$

The numerator extends to a linear function, but the denominator contains square roots of the coordinates. But it still has the good property that it has the minimum number of critical points. A more general question is how the geometry and metric of the projective space can be used to obtain a deeper understanding.

It seems also worth adding that Theorem 1 suggests several further developments in the same spirit.

First, one can obtain similar descriptions of extremals of the area under different constraints. The most obvious option is to fix the sum of certain powers of the side lengths. In other words, one looks for extremals of the area under the condition that

$$
\sum\left(a_{i}\right)^{p}=\text { const, }
$$

where $p$ is a fixed positive number. For symmetry reasons, regular stars and complete folds are critical in this case too. However, it is not obvious that there are no other extremals, since the geometric reasoning used for fixed perimeter is not directly applicable. For $p=2$, a rigorous analytic proof of this fact is given in [6] without discussing the nondegeneracy and Morse indices of stars. Perhaps, this case fits better in the framework of the Veronese embedding. Moreover, it is not difficult to verify that the same method of proof is applicable for any positive $p$.

Finally, in line with the general idea of duality in the calculus of variations, one may consider a dual problem by fixing the values of the area and looking for extremals of the perimeter. Some conclusions and lines of research are immediate. For example, it is easy to verify that generically the critical points of $A$ with $\mathcal{P}$ fixed are the same as the critical points of $\mathcal{P}$ with $A$ fixed. One can also derive the Morse index formula for the dual problem from our Theorem 1.

\section{ACKNOWLEDGMENTS}

It is our pleasure to acknowledge the hospitality and excellent working conditions of CIRM, Luminy, where this paper was initiated as a "Research in Pairs" project. This work is supported by the RFBR grant 17-01-00128 and by the Presidium of RAS program "New Methods of Mathematical Modelling in Nonlinear Dynamical Systems" (grant 08-04).

\section{REFERENCES}

1. A. A. Agrachev, D. Pallaschke, and S. Scholtes, "On Morse theory for piecewise smooth functions," J. Dynam. Control Systems, 3, No. 4, 449-469 (1997).

2. V. Blåsjö, "The isoperimetric problem," Amer. Math. Monthly, 112, No. 6, 525-566 (2005). 
3. T. E. Cecil and P. J. Ryan, Tight and Taut Immersions of Manifolds, Pitman, Boston (1985).

4. F. H. Clarke, "Generalized gradients and applications," Trans. Amer. Math. Soc., 205, 247-262 (1975).

5. J. Gordon, G. Panina, and Y. Teplitskaya, "Polygons with prescribed edge slopes: configuration space and extremal points of perimeter," Beitr. Algebra Geom., 60, No. 1, 1-15 (2019).

6. J. C. Leger, "Aire, périmètre et polygones cocycliques," arXiv:1805.05423 (2018).

7. A.-M. Legendre, Éléments de géométrie, 14e edition, Firmin Didot frères (1841).

8. M. Kapovich and J. Millson, "On the moduli space of polygons in the Euclidean plane," J. Differential Geom., 42, No. 1, 133-164 (1995).

9. G. Khimshiashvili and G. Panina, "Cyclic polygons are critical points of area," Zap. Nauchn. Semin. POMI, 360, No. 8, 238-245 (2008).

10. G. Panina and A. Zhukova, "Morse index of a cyclic polygon," Cent. Eur. J. Math., 9, No. 2, 364-377 (2011).

11. A. Zhukova, "Morse index of a cyclic polygon II," St. Petersburg Math. J., 24, 461-474 (2013). 